\title{
Technique for measuring the two-port scattering matrix of a plasma current drive antenna during high power operation
}

\author{
P. H. Probert ${ }^{\text {a) }}$ and R. P. Majeski ${ }^{\text {b) }}$ \\ Department of Nuclear Engineering, Engineering Physics, and Engineering Mechanics, \\ University of Wisconsin, Madison, Wisconsin 53705
}

(Received 14 August 1996; accepted for publication 4 November 1996)

\begin{abstract}
We describe a technique to routinely measure the scattering matrix of a radio frequency (rf) current drive antenna in a magnetic fusion experiment during high power operation. This technique can be used under any conditions of phase or amplitude of excitation. It involves modulation of the phase of the rf voltage applied to each of the antenna ports by a few degrees, measurement of the complex forward and reflected voltages, detection of the phase modulation, and solution of a $2 \times 2$ matrix problem to yield the scattering matrix. Further calculation then yields the antenna's impedance matrix. Because it involves only a small modulation, this method can be used to routinely monitor matching, decoupling, or plasma loading and to provide input to tuning calculations so that the matching and phasing conditions can be maintained. We show results for the case of $400 \mathrm{~kW}$ Alfvèn wave current drive experiments on the Phaedrus-T tokamak. We also show how the results can be used to tune the antenna. (C) 1997 American Institute of Physics. [S0034-6748(97)01602-X]
\end{abstract}

\section{INTRODUCTION}

Magnetic fusion research devices such as tokamaks often employ radio frequency (rf) systems in the ion cyclotron, Alfvèn, or lower-hybrid frequency ranges to drive plasma current. Such systems usually employ antennas with two or more feed points that must be driven such that the currents or voltages on the plasma-facing antenna elements are in a controlled phase relationship so that directional waves can be launched. Further, any reflections or cross coupling of power must be controlled in order to maximize the coupled power while simultaneously protecting the transmitters.

Most current drive antennas are connected to the transmitters through matching circuits which can consist of tuning elements such as stubs, variable capacitors, or inductors, and variable length lines (many lower-hybrid systems and some ion cyclotron systems avoid the matching circuits and tolerate any reflected power by some other means). In addition, a method of decoupling using power compensators, ${ }^{1}$ hybrid couplers, ${ }^{2}$ or inductive decouplers ${ }^{3}$ is usually employed to cancel the coupling between the plasma-facing antenna elements. In all these schemes, some measurement technique is required to provide knowledge of the circuit conditions so that the various adjustable tuning and decoupling elements can be properly set.

Often workers have resorted to a combination of low power measurements, estimations based on incomplete scattering measurements, and trial and error methods to arrive at a suitable tuning. These methods have several drawbacks. (1) Tuning at low power can be difficult if the plasma properties are a function of the rf power. For example, modifications to the plasma edge density profile and the release of impurities have been observed in high power heating in the ion cyclotron range of frequencies (ICRF), ${ }^{4,5}$ which can dramatically

\footnotetext{
${ }^{a)}$ Present address: Torsatron-Stellarator Laboratory, Dept. of Electrical and Computer Engineering, University of Wisconsin, Madison, WI; Electronic mail: pprobert@facstaff.wisc.edu

${ }^{b)}$ Present address: Princeton Plasma Physics Laboratory, Princeton, NJ.
}

affect the antenna loading. Ponderomotive effects can also alter the density local to the antenna at high power. (2) Estimates often involve simplifying assumptions about such things as the symmetry of the scattering matrix or the phase of the coupling process (e.g., that the coupling between antenna elements is purely a mutual inductance, and that waves in the plasma do not shift the phase of the coupling). (3) Many plasma discharges can be required for trial and error methods, wasting valuable resources and limiting the number of experimental scans that can be accomplished. What we describe here is a method of measuring the full complex scattering matrix of a two input antenna system during routine, high power operation, and we show the results of using this method on the Phaedrus-T tokamak.

\section{SCATTERING MATRIX MEASUREMENT}

Let us represent the inputs to a two-port antenna system as terminals of transmission lines of characteristic impedance $Z_{0}$, and label these terminals A and B. We assume that there are signals of only one frequency $\omega$, and that we can represent real sinusoidal signals

$$
\nu(t)=\nu_{0} \cos (\omega t+\varphi)=\operatorname{Re}\left(V e^{j \omega t}\right),
$$

where the complex number $V \equiv \nu_{0} e^{j \varphi}$ is the phasor representation of the signal. Then we represent the electrical states of these inputs (ports) by the complex forward and reflected voltages

$$
\mathbf{V}_{F} \equiv\left[\begin{array}{c}
V_{F A} \\
V_{F B}
\end{array}\right] \text { and } \mathbf{V}_{R} \equiv\left[\begin{array}{c}
V_{R A} \\
V_{R B}
\end{array}\right] \text {. }
$$

Then we define the scattering matrix

$$
\mathbf{S} \equiv\left[\begin{array}{ll}
S_{A A} & S_{A B} \\
S_{B A} & S_{B B}
\end{array}\right]
$$


such that $\mathbf{V}_{R}=\mathbf{S V}_{F}$. Transmission line theory relates the voltages and currents at the terminals by the formulas $V_{X}$ $=V_{F X}+V_{R X}$ and $I_{X}=\left(V_{F X}-V_{R X}\right) / Z_{0}($ where $X$ is A or B), or, in matrix notation,

$$
\mathbf{V}=(\mathbf{1}+\mathbf{S}) \mathbf{V}_{F}
$$

and

$$
\mathbf{I}=(\mathbf{1}-\mathbf{S}) \mathbf{V}_{F} / Z_{0},
$$

where $\mathbf{1}$ is the identity matrix.

Now the scattering matrix contains four complex numbers, and so in general a single measurement in which we apply known forward voltages to the inputs and measure the reflections cannot yield sufficient information to deduce the scattering matrix. Instead, if we obtain two data sets, labeled 1 and 2 , then a set of matrix equations results as

$$
\mathbf{V}_{R 1}=\mathbf{S V}_{F 1}
$$

and

$$
\mathbf{V}_{R 2}=\mathbf{S V}_{F 2} \text {. }
$$

If we form the matrices

$$
\mathbf{V}_{\mathbf{R}} \equiv\left[\begin{array}{ll}
V_{R A 1} & V_{R A 2} \\
V_{R B 1} & V_{R B 2}
\end{array}\right] \text { and } \mathbf{V}_{\mathbf{F}} \equiv\left[\begin{array}{ll}
V_{F A 1} & V_{F A 2} \\
V_{F B 1} & V_{F B 2}
\end{array}\right] \text {, }
$$

then we can solve the system, giving $\mathbf{S}=\mathbf{V}_{\mathbf{R}} \mathbf{V}_{\mathbf{F}}^{-1}$ provided that the inverse of $\mathbf{V}_{\mathbf{F}}$ exists, which is tantamount to requiring that the two data sets are really different measurements, i.e., that the forward voltages applied in the two cases are linearly independent.

The foremost result of this article is to demonstrate a convenient way of obtaining these two data sets. We note that a method of experimentally determining the scattering matrix reported by Lamalle et al. ${ }^{6}$ on the Joint European Torus (JET) device is qualitatively similar to our method. Rather than obtaining data sets at two discrete conditions, however, they obtain a data set in which the phase is continuously varied, and an estimate of the scattering matrix is calculated from a least-squares fitting procedure. Likewise, a method employed by Kazumi et al. ${ }^{7}$ on the JFT-2M device uses a fitting procedure to estimate the antenna impedance matrix using data sets from several shots. In both of these techniques the shots in which the measurements are made differ from the intended high power, controlled phase shots that are eventually desired, and so the measured loading may not be correct. In the work here we show a method that allows these measurements to be made without the need for a series of special shots. Furthermore, in our method we do not employ a fitting procedure to estimate the scattering matrix, we calculate it directly.

\section{PHAEDRUS-T TOKAMAK}

The Phaedrus-T tokamak is a medium-sized tokamak dedicated to rf physics and current drive studies. Phaedrus-T, the rf system and antennas, and the major results concerning Alfvèn wave heating and current drive have been discussed elsewhere. ${ }^{3,8-11}$ Briefly, it has a maximum magnetic field of $1 \mathrm{~T}$, a major radius of $0.92 \mathrm{~m}$, a minor radius of $0.26 \mathrm{~m}$, a maximum density of $3 \times 10^{19} \mathrm{~m}^{-3}$, and a pulse length of 180

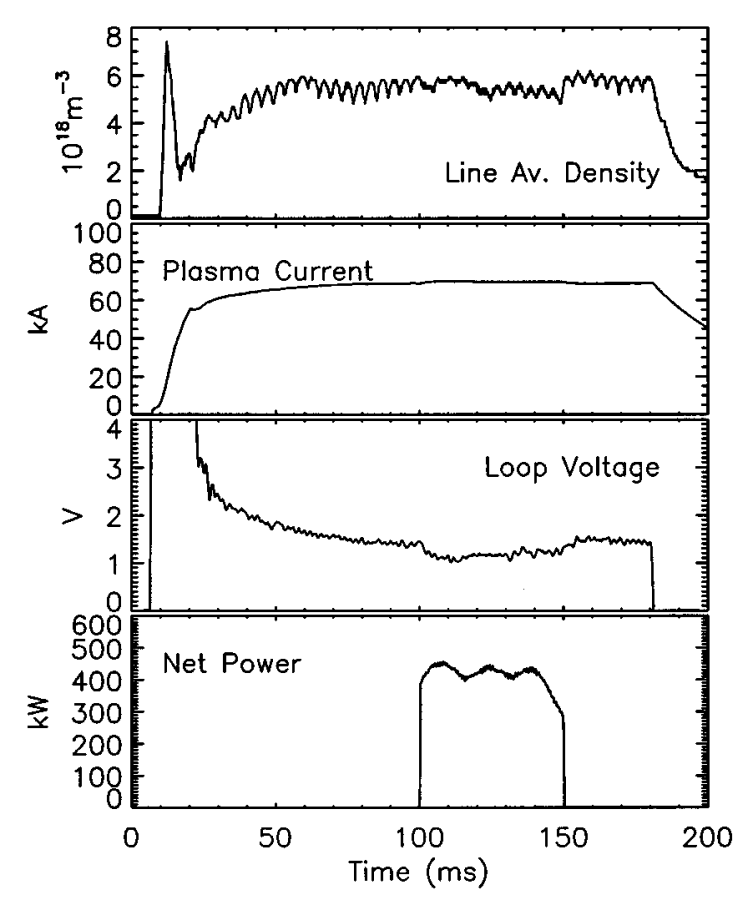

FIG. 1. Line averaged plasma density from a vertical central chord as measured with a $140 \mathrm{GHz}$ interferometer, plasma current, loop voltage, and net (forward minus reflected) rf power during a typical $7 \mathrm{MHz}$ Alfvèn wave current drive experiment. The toroidal magnetic field was $0.7 \mathrm{~T}$ for this shot.

ms. A plot of the main plasma diagnostics for a typical shot is shown in Fig. 1. Here the plasma parameters were reduced somewhat during an investigation of current drive at a toroidal magnetic field on axis of $0.7 \mathrm{~T}$. The slight reduction in the loop voltage during the rf pulse is due to current drive effects.

In the range of 4-20 MHz rf is available from two transmitters to a maximum power of about $1 \mathrm{MW}$. All of the results reported here concern the use of a low-field-side two strap antenna with a spacing of $14 \mathrm{~cm}$, as used in our current drive studies. ${ }^{9}$ An adjustable inductive decoupler is connected to reduce the mutual inductance of the two straps. ${ }^{3} \mathrm{~A}$ block diagram of the rf system is shown in Fig. 2. A key feature of the rf system is that, as shown, the two transmitters are driven indendently by low power oscillators that can be phase modulated in an arbitrary way. We also show a pair of directional couplers on the inputs to the matching circuits that provide all the data used here. These units have a coupling of about $-70 \mathrm{~dB}$ and a directivity of about $40 \mathrm{~dB}$.

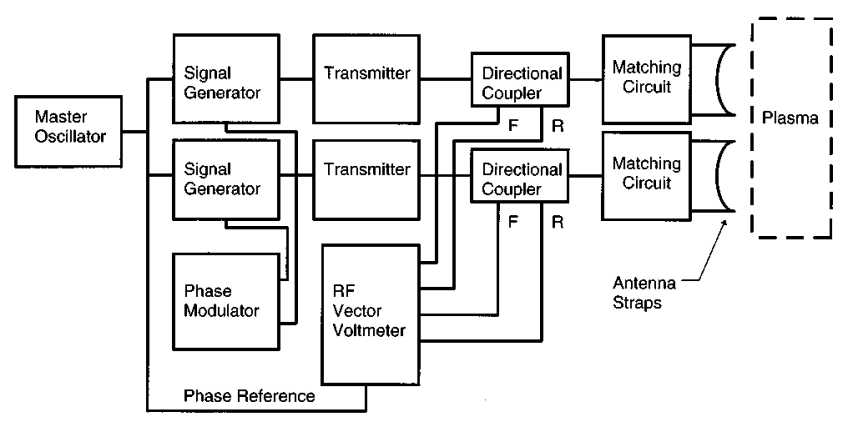

FIG. 2. Block diagram of the two channel rf system used in Phaedrus-T. 


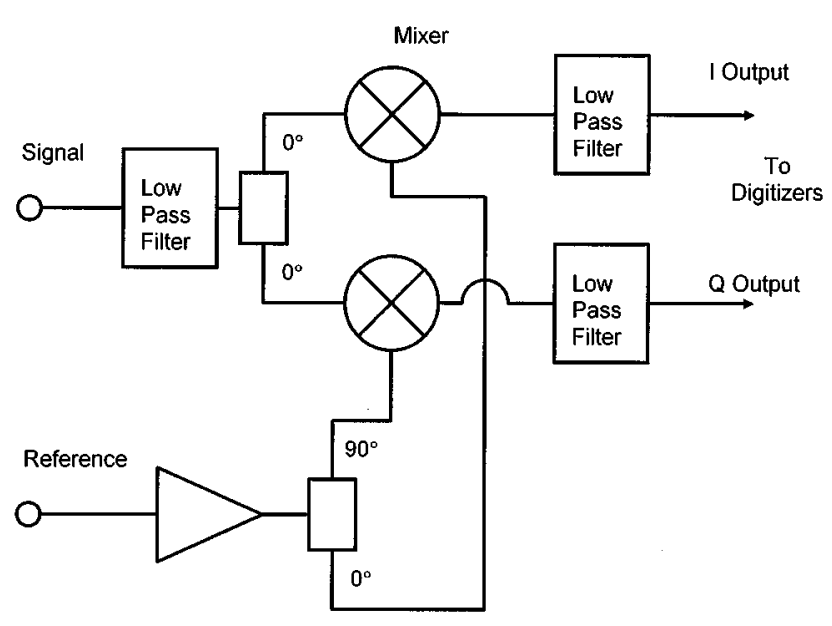

FIG. 3. Simplified schematic of a channel of our vector voltmeter for measuring the amplitude and phase of signals from the directional couplers.

They are calibrated at low power using a Hewlett Packard $8508 \mathrm{~B}$ vector voltmeter.

\section{IV. rf MEASUREMENTS}

An essential element in the apparatus is the vector voltmeter that is shown in the aforementioned block diagram, Fig. 2. We describe this unit in some detail here because in our experience the standard methods for measuring rf signals in fusion experiments can introduce errors large enough to significantly degrade the accuracy of the calculated scattering matrix measurements, due to the small phase modulations we employ here and the extensive calculations performed on the data. This unit measures the phase and amplitude of the directional coupler signals. A simplified diagram that shows one channel of this vector voltmeter is shown in Fig. 3. The reference, which is generated by the transmitter system's master oscillator, is common to all the channels and defines the phase. The mixers used are passive double balanced diode mixers, and the reference signal driving the local oscillator inputs is set at a high enough level to saturate the mixers. An important element is the low-pass filter at the input of the vector voltmeter. Although the double balanced construction of the mixers rejects the second harmonic effectively, the third harmonic can interfere strongly with the measurement. We have found that at high power a significant amount of third harmonic energy is generated by the transmitters and the plasma and appears at the output of the directional couplers. This is made worse by the directional couplers, which have a sensitivity that increases linearly with frequency. Thus the low-pass filter at the input must be selected to be effective at third and higher harmonics.

Through careful design the dynamic range of the vector voltmeter is very nearly that of the 12 bit digitizers that record the output signal. This makes it possible to leave the system unchanged during low and high power measurements.

The outputs $I$ and $Q$ shown in Fig. 3 are named, as is common in $\mathrm{rf}$ practice, to mean In phase and Quadrature phase. In terms of the general rf signal [Eq. (1)], the $I$ output is proportional to $\nu_{0} \cos (\varphi)$ and the $Q$ output is proportional to $\nu_{0} \sin (\varphi)$. The low-pass filters shown at the outputs of the vector voltmeter are designed to block all signals at the rf frequency and to prevent aliasing in the digitizing process. In the data we report here these filters have a $20 \mathrm{kHz}$ cutoff frequency, and digitizing is done at a 100 kilosamples/s rate. All other manipulations of the signals are done with software, where the signal from the vector voltmeter is represented as a complex phasor function of time $V(t)$ that varies slowly with respect to both the rf frequency and the sample rate. In order to increase the accuracy of the measurements, we calibrate the vector voltmeter by applying the same swept phase signal to all channels simultaneously and recording the output. Using this data, we calculate corrections for the relative phase offset of the channels, the relative gains of all the $I$ and $Q$ outputs, and any error in the phase angles of the outputs of the $90^{\circ}$ splitter shown in the reference leg in Fig. 3. After applying these corrections we can obtain an accuracy of abut $1 \%$ in amplitude and about $1^{\circ}$ in phase for most signals.

\section{OBTAINING THE DATA SETS}

The obvious first choice in obtaining the data sets [Eq. (3)] is to take two separate shots in which some property of the rf was altered between the shots. For instance, one can take a shot in which rf is applied to antenna port A, and then one with $\mathrm{B}$ only. Better, to maintain constant total power, shots A and B can differ only in the phase between the excitation to the two ports. To minimize the number of shots involved, one can refine the technique slightly by "chopping" the phase of the drive to one of the input ports one or more times during the shot so that both data sets are obtained in a single shot. In Fig. 4 we show some of the rf signals from such a shot in Phaedrus-T, where the phase of the B channel was chopped by a large amount with a period of 10 ms. We then obtain data set 1 by averaging data over a half-period in which the phase is constant at one value, and obtain data set 2 by averaging over the next half-period. Later, in Sec. VII, we show the impedance matrix derived from this data.

The main result of this article is based on yet a further refinement to this chopping technique. There are two steps in this refinement: (1) reduce the phase modulation from a large swing in the phase to just a few degrees; (2) increase the frequency of the modulation to $1 \mathrm{kHz}$ (in principle this could be increased many times further); (3) modulate the signal generators that drive both transmitter channels by a sine wave phase modulation, where channel B's phase modulation is delayed in time by $1 / 4$ of the modulation period relative to the modulation of channel A; (4) obtain data sets 1 and 2 by extracting the modulated components of the signals from the raw data using a numerical demodulation technique.

\section{DEMODULATING THE SIGNALS}

Let us assume we have modulated the drive to the $\mathrm{A}$ transmitter so that it has the form given in Eq. (1) with the phase $\varphi_{A}=\varphi_{A D C}+\varphi_{0} \cos \left(\omega_{\bmod } t\right)$, where the modulation frequency $\omega_{\text {mod }}$ corresponds in our case to $1 \mathrm{kHz}$, much less 


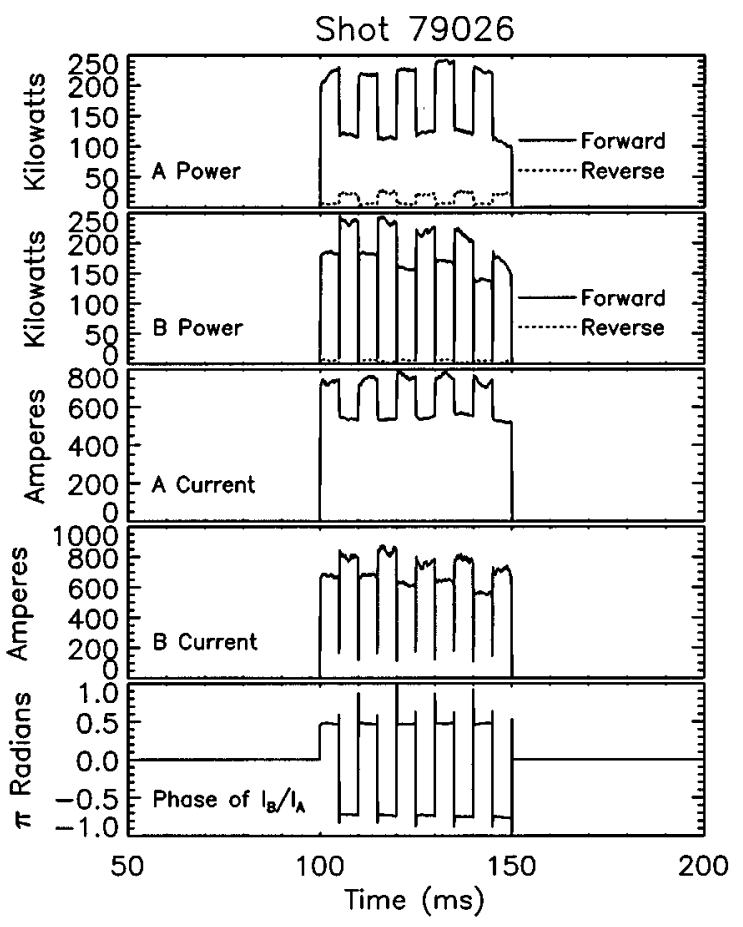

FIG. 4. rf powers and antenna currents during a shot in which the phase of the drive to the B channel was "chopped" by a large amount with a period of $10 \mathrm{~ms}$. The two data sets necessary for calculation of the scattering matrix can be obtained by using data from such a shot by looking at two times when the phase has distinctly different values. The large variation of the A signals with phase, although only the phase of the B channel is being varied, is a reflection of the fact that, although the decoupler is cancelling most of the cross coupling, there is still enough residual coupling to have a noticeable effect. This effect is made worse by our transmitters (grounded grid triode final stages) which not only do not absorb reflections but seem to amplify them, as the traces of channel A's forward and reflected powers show.

than the rf frequency of $7 \mathrm{MHz}$. The modulation amplitude $\varphi_{0}$ corresponds to a peak to peak phase shift of the rf signal of $10^{\circ}$ or less. Likewise, the drive to $\mathrm{B}$ is modulated with $\varphi_{B}=\varphi_{B D C}+\varphi_{0} \sin \left(\omega_{\text {mod }} t\right)$. Our demodulation technique then attempts to decompose all the detected rf signals from the directional couplers into a sum of signals proportional to these two drives. Let a general signal's phasor be represented by $V=V^{A} \exp \left[j \varphi_{0} \cos \left(\omega_{\text {mod }} t\right)\right]+V^{B} \exp \left[j \varphi_{0} \sin \left(\omega_{\text {mod }} t\right)\right]$, where we use the superscripts to denote that part of the signal that carries the phase modulation of channel A or B. Then we can calculate

$$
V^{A}\left(t^{\prime}\right)=\operatorname{const} \times \int_{N} \cos \left(\omega_{\bmod } t\right) V d t
$$

where we assume that $\varphi_{0} \ll 1$ and where the integral is over an integer $N$ cycles of the modulation centered on the time $t^{\prime}$. The value of the constant multiplier, const $=2 / j N \varphi_{0}$ is not important in the subsequent calculations since only ratios are taken. The value of $V^{B}$ is calculated with $\sin ()$ instead of $\cos ()$ in the kernel of the integral. Finally, we apply this demodulation formula to the four signals from the directional couplers to obtain our matrices of scattering data:

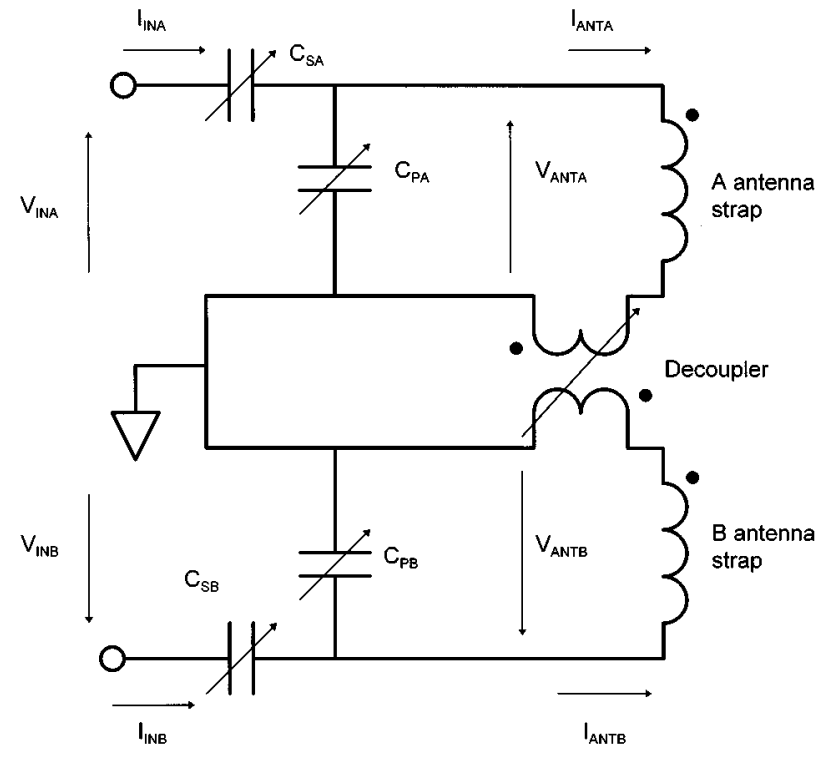

FIG. 5. Electrical schematic of the antenna and matching circuits. The A and B straps are inductively coupled because they are parallel and $14 \mathrm{~cm}$ apart, and the decoupler cancels this coupling by introducing a mutual inductance in the opposite sense (as indicated by the dots). The capacitors are vacuum variable units that can be tuned on a shot to shot basis by manually rotating their dials. A more accurate circuit model is to include stray reactances (not shown). In particular, we have found that stray capacitance from the input terminal to ground and stray inductance in series with the $\mathbf{C}_{p}$ capacitors is important in our circuit models (see the text).

$$
\mathbf{V}_{\mathbf{R}}=\left[\begin{array}{cc}
V_{R A}^{A} & V_{R A}^{B} \\
V_{R B}^{A} & V_{R B}^{B}
\end{array}\right]
$$

and

$$
\mathbf{V}_{\mathbf{F}}=\left[\begin{array}{cc}
V_{F A}^{A} & V_{F A}^{B} \\
V_{F B}^{A} & V_{F B}^{B}
\end{array}\right],
$$

from which we can compute the scattering matrix, as earlier, with $\mathbf{S}=\mathbf{V}_{\mathbf{R}} \mathbf{V}_{\mathbf{F}}^{-1}$. By using distinct modulations we are assured that the inverse of the forward signal matrix exists. We note that in the case where the forward voltage signal on the high power transmission lines is strictly proportional to the drive signal the matrix $\mathbf{V}_{\mathbf{F}}$ is diagonal and inversion is trivial. That is, if there is no amount of the modulation of the B channel appearing on the A forward voltage and vice versa, then identification of the origin of the scattered signals is easy. In our case, this is not strictly true. Whenever there is cross-coupled power in the antenna and the transmitter's output is not matched (common in high power circuits), then there is some contamination of each forward signal with the modulation of the other channel due to reflection of the cross-coupled reverse signal from the transmitter. By measuring and inverting the full matrix this contamination is eliminated at the cost of some computational complexity. Although not of concern for us, it might be important in a future application where the scattering matrix must be computed in real time in a feedback loop tuning system. 


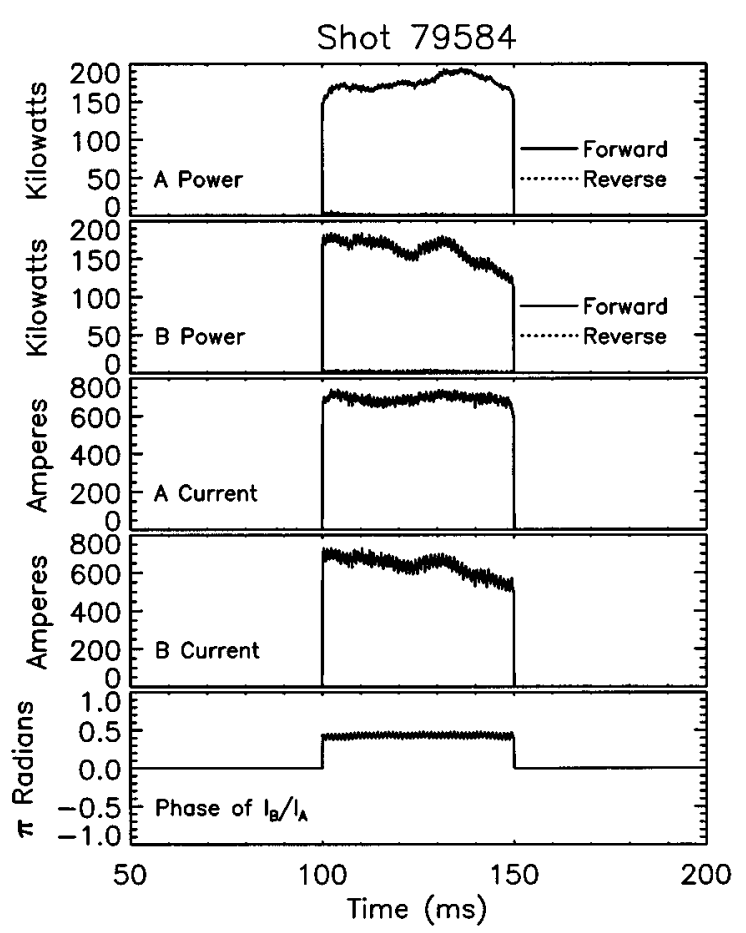

FIG. 6. rf data for a shot in which phase modulation was applied to both channels (see the text). The modulation frequency was $1 \mathrm{kHz}$; the peak to peak phase deviation on either channel was $10^{\circ}$.

\section{IMPEDANCE MATRIX CALCULATION}

Scattering matrices, though a complete description from the point of view of the transmitters, are not as interesting to the experimentalist as phenomena occurring at the antenna straps themselves. In Fig. 5 we show an electrical schematic of our two strap antenna. On the left of the diagram the input voltages and currents are shown, which can be calculated from the scattering data using Eq. (2). One can then define and calculate an antenna impedance matrix $Z_{\text {ant }}$ such that

$$
\left[\begin{array}{c}
V_{\mathrm{ant} A} \\
V_{\mathrm{ant} B}
\end{array}\right]=\mathbf{Z}_{\mathrm{ant}}\left[\begin{array}{c}
I_{\mathrm{ant} A} \\
I_{\mathrm{ant} B}
\end{array}\right] .
$$

It is a simple matter to calculate the strap voltages and currents using circuit equations if the matching circuit capacitances are known. Given the two data sets, $\mathbf{V}_{\mathbf{R}}$ and $\mathbf{V}_{\mathbf{F}}$, one can calculate the input voltages and currents $V_{\text {inA }}$, $I_{\mathrm{in} A}, V_{\mathrm{in} B}, I_{\mathrm{in} B}$ and then solve for the outputs. For our simplified circuit, for example, we get $V_{\text {ant }}=I_{\text {in }} / j \omega C_{s}+V_{\text {in }}$, $I_{\text {ant }}=I_{\text {in }}-V_{\text {ant }} j \omega C_{p}$. Then, for these two data sets we can construct matrices

$$
\mathbf{V}_{\text {ant }}=\left[\begin{array}{ll}
V_{\mathrm{ant} A 1} & V_{\mathrm{ant} A 2} \\
V_{\mathrm{ant} B 1} & V_{\mathrm{ant} B 2}
\end{array}\right], \quad \mathbf{I}_{\mathrm{ant}}=\left[\begin{array}{ll}
I_{\mathrm{ant} A 1} & I_{\mathrm{ant} A 2} \\
I_{\mathrm{ant} B 1} & I_{\mathrm{ant} B 2}
\end{array}\right],
$$

and then solve for the strap impedance matrix to get $\mathbf{Z}_{\text {strap }}$ $=\mathbf{V}_{\mathrm{ant}} \mathbf{I}_{\mathrm{ant}}^{-1}$. If the original two data sets are unavailable but the scattering matrix is known, this calculation can proceed by setting

$$
\mathbf{V}_{\mathbf{F}}=\left[\begin{array}{ll}
1 & 0 \\
0 & 1
\end{array}\right], \text { then } \mathbf{V}_{\mathbf{R}}=\mathbf{S V}_{\mathbf{F}}=\mathbf{S} .
$$

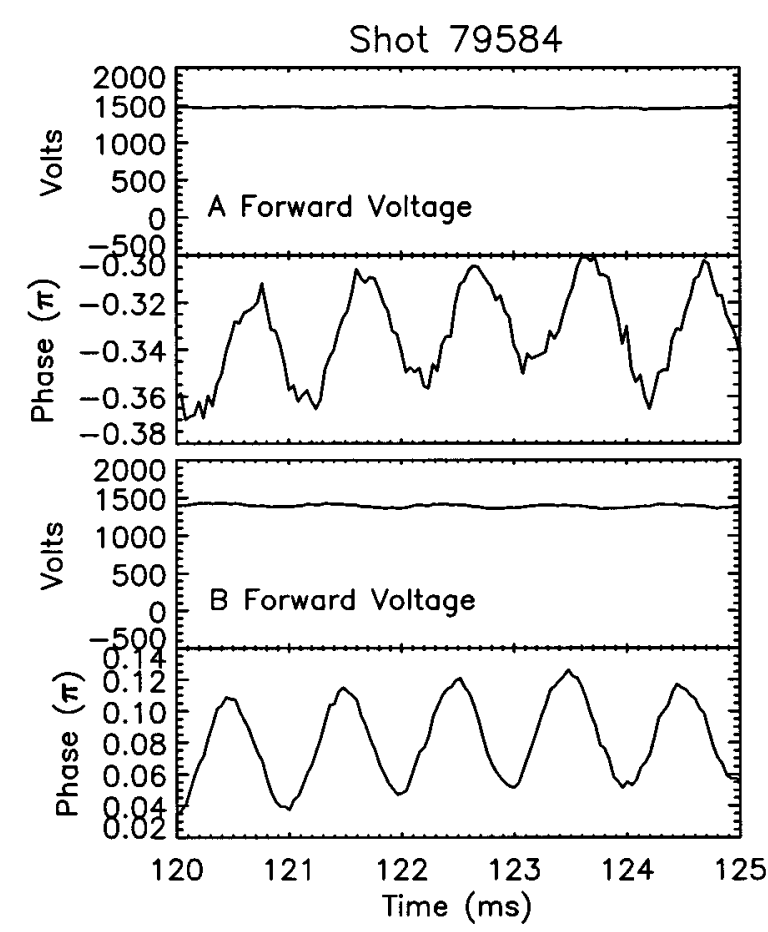

FIG. 7. Data from the same shot as that of Fig. 6. Here we show the magnitude and phase of the forward voltages on the transmission lines of the two channels on an expanded time scale to reveal the modulation.

In our case we have found that better results can be obtained by taking into account several stray reactances not shown, such as a stray capacitance from the input terminal to ground and the stray series inductances of the parallel capacitors $\mathbf{C}_{\mathrm{PA}} \mathbf{C}_{\mathrm{PB}}$. Returning to the example data shown in Fig. 4 for our shot No 79026, we calculate the impedance matrix, using the data just before and just after the phase transition at 120 ms for our two data sets, to be

$$
\mathbf{Z}_{\mathrm{ant}}=\left[\begin{array}{cc}
0.372+j 20.8 & 0.09+j 0.009 \\
0.106+j 0.023 & 0.355+j 20.1
\end{array}\right] .
$$

On the diagonal we see the real parts are the loading, here mostly due to the plasma (about $0.08 \Omega$ can be attributed to the resistance of the copper straps) where the imaginary parts are the self-inductances of the straps. The offdiagonal elements represent the coupling between straps, either purely inductively or through plasma waves. By coupling through slower plasma waves the coupling can gain a phase shift, giving rise to the real parts of the off-diagonal elements. Were this measurement made without the inductive decoupler, the imaginary parts of the off-diagonal elements would be about $1 \Omega$ in vacuum. The decoupler removes most of this fixed imaginary part of the off-diagonal elements. With plasma present, the off-diagonal elements change by about $10 \%$, and some real part (about $10 \%$ of the $1 \Omega$ of vacuum coupling) is introduced. This remaining coupling, especially the real parts, gives rise to the large changes with phase of the channel A and B forward powers in Fig. 4, which, in the uncoupled case, should be flat. 
Z Matrix, Shot 79584
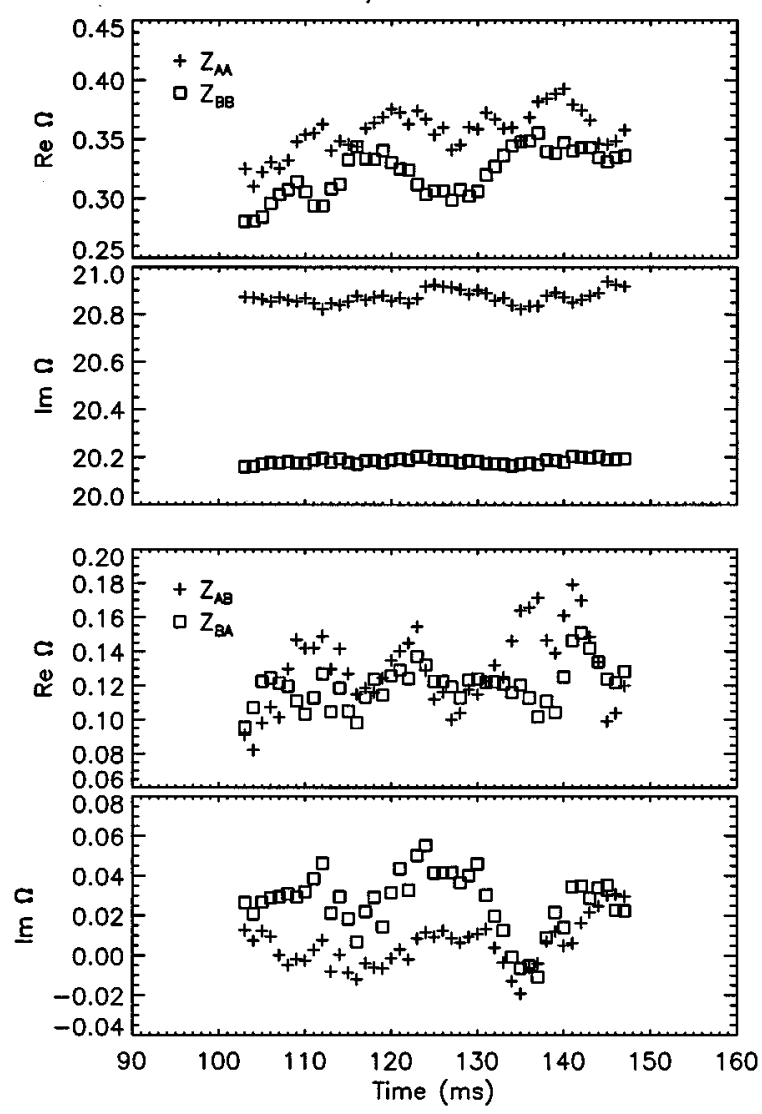

FIG. 8. The results of calculating the impedance matrix from the data from the shot in Fig. 6. Here a value is generated for every cycle of the phase modulation. Most of the variations seen during the shot can be attributed to motions of the plasma column relative to the antenna.

\section{EXPERIMENTAL TESTS}

We show in Fig. 6 the rf diagnostics during a pulse in which the phase modulation technique was used with a modulation amplitude of $5^{\circ}$ (peak to peak modulation $10^{\circ}$ ). In Fig. 7 the phase and amplitude of the directional coupler forward signals are shown on an expanded scale, revealing both the modulation and the amount of noise typically present. In Fig. 8 we show the output of the analysis of a shot in the form of plots of the antenna impedance matrix elements as functions of time.

The noise level is important because we would like to be able to use the least amount of modulation possible so that the experiments are not affected. To assess the tolerance of the technique to noise, we performed a scan in which the phase modulation was reduced from shot to shot. In Fig. 9 we show the results of this scan that reveal that in our experiments we need at least $1^{\circ}$ of modulation to stay above the noise. This is the level at which plasma effects induce phase shifts in the reflected voltage signal that are comparable to our modulation phase shifts.

Finally, to be sure that the phase modulation technique yields the same answers as the simpler "chopping" method, we performed a scan in which we varied the decoupler setting from shot to shot. The effect of varying the decoupler setting is to change the mutual inductance between the an-
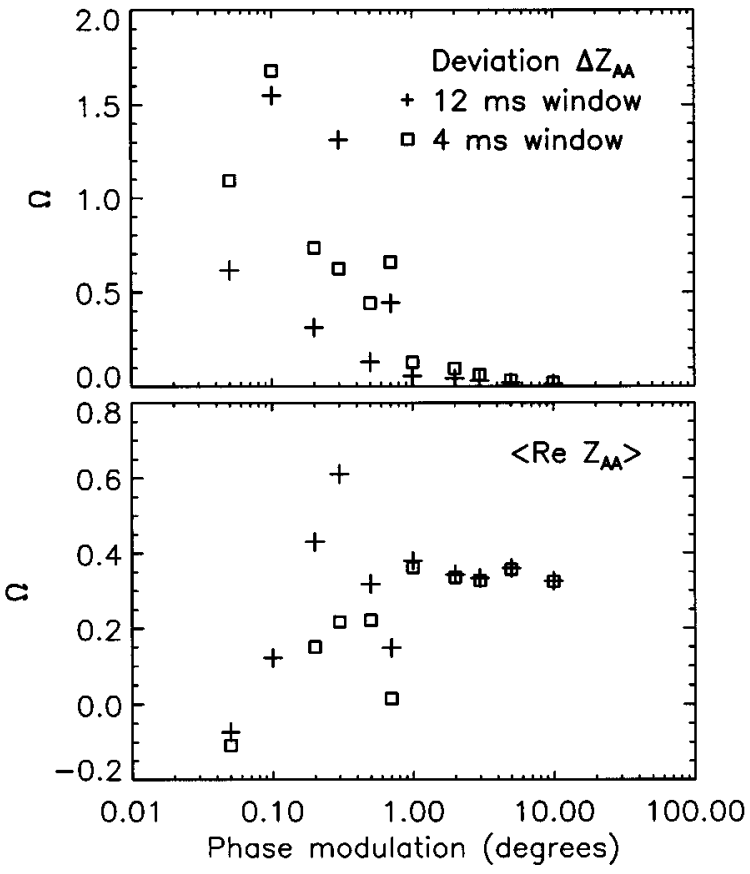

FIG. 9. The results of a scan in which the amount of phase modulation was varied from shot to shot. At the top is the magnitude of the root mean square difference of the $Z_{A A}$ values from the average of all the values during the shot, reflecting the amount by which this value changes during the shot. At the bottom is the average of the real part of this value over the whole shot. The meaning of the window times (12 and $4 \mathrm{~ms}$ ) is that the value of $N$ in Eq. (4) is either 12 or 4 in the demodulation process.

tenna straps, which is seen in the imaginary parts of the off-diagonal elements of the antenna impedance matrix. The results of this scan are shown in Fig. 10. We see that the results are in close agreement.

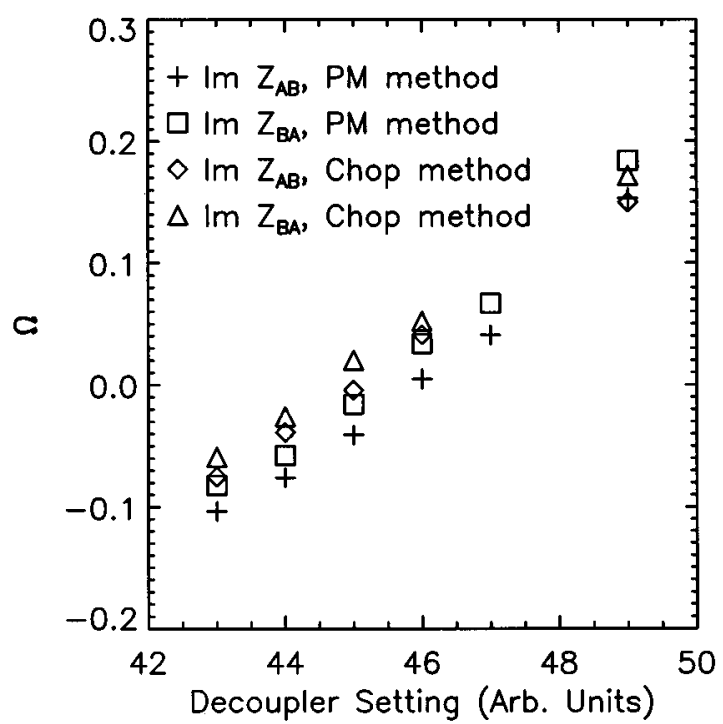

FIG. 10. Imaginary parts of the off-diagonal impedance matrix elements measured during a scan in which the mutual inductance of the decoupler was changed from shot to shot. In the "chop" method the measurement was made using large phase changes as in Fig. 4, and in the "PM" method the measurement was taken using phase modulation with a peak to peak modulation of $10^{\circ}$, as in Fig. 6. A decoupler setting of 45 , where the mutual inductance is roughly 0 , is where we normally operate the antenna. 


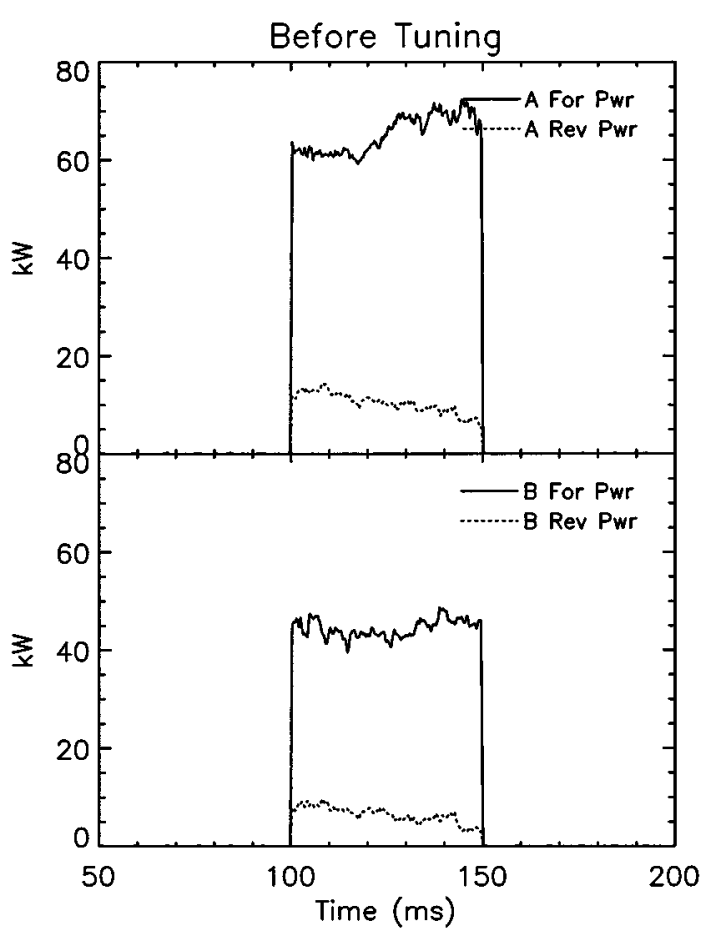

FIG. 11. Forward and reflected powers for a shot taken after some antenna work such that the impedance matrix had changed and we no longer had a good tuning. The shot was taken at reduced power to avoid tripping the reflected power interlocks on our transmitter.

\section{USING THE IMPEDANCE MATRIX MEASUREMENTS IN THE TUNING PROCESS}

To validate the measurement process described above, we now show how the results of the impedance matrix measurements can be successfully used in tuning the antenna.

We mentioned earlier that, although the decoupler removes a great deal of the mutual inductance between the antenna straps, there is still a residual amount that cannot be canceled because of a phase shift in the coupling. There is, however, an opportunity to keep this coupled power from finding its way back to the transmitters. The idea is that, if the matching circuits are slightly detuned in the proper way, then we can create a reflection that will destructively interfere with the cross-coupled signal. By having a good measurement of the impedance matrix, plus an accurate model of the matching circuits, we should have sufficient information to solve this problem. By implementing a numerical routine based on a Newton's method root finder, we are able to calculate the matching circuit settings necessary to perform antenna tuning. The basic strategy is to create a vector of six quantities which we wish to make zero. These are the real and imaginary parts of the reverse voltage on the two transmission lines, the difference between the two antenna current magnitudes, and the difference between the relative antenna current phase and the desired relative antenna current phase. To find the zero of this vector, we have four capacitor settings and two transmitter drive settings (phase and relative amplitude) to vary. We use a circuit model involving the matching circuits (with all the stray reactances mentioned above included) connected to a theoretical two-port network

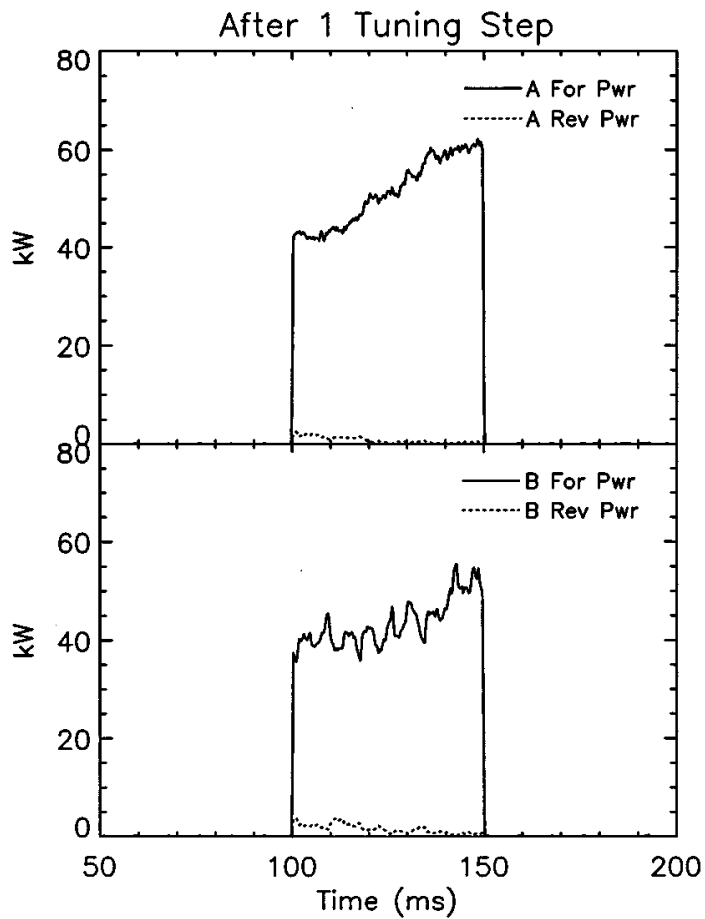

FIG. 12. Data for a shot taken several shots after the shot shown in Fig. 11. Between these shots the impedance matrix was measured once, the numerical tuning algorithm was executed using this data once, and the recommended matching circuit capacitor settings were applied.

with an impedance matrix equal to that measured. Provided the root exists and that we can make an initial guess close enough to the solution, convergence is rapid. To demonstrate this, we show in Fig. 11 the results of a shot taken shortly after the antenna had undergone some modifications so that we did not have a good tuning. In Fig. 12 we show data from a few shots later; after the impedance matrix was measured once, our tuning algorithm was run once, and the recommended capacitor settings were dialed in. We see that the reflected powers were significantly reduced. Usually the initial guess for a tuning can be obtained by tuning the antenna to resonance in a vacuum at low power. Then, one shot is taken at reduced power with plasma in which the impedance matrix is measured. After running the tuning algorithm and dialing in the new tuning, the antenna is ready for high power operation. One more impedance matrix measurement and tuning at high power will result in almost negligible reflected power provided the plasma conditions are not changed.

\section{IDEAS FOR REFINEMENT}

There is room for further refinement of our technique. For example, the method could be extended to an antenna with more than two ports by using a more sophisticated modulation technique, such as one employing multiple modulation frequencies. Also, if one needed to reduce the amount of phase modulation below the $1^{\circ}$ limit observed here, it might be possible to employ higher modulation frequencies where there may be less plasma noise. Another possibility would be to use noise resistant modulation, such as a 
pulse code modulation scheme. Such a scheme would make the extension to more than two ports very easy as well.

In at least one fusion experiment, the JET device, ${ }^{1,12}$ the antenna tuning is included in a sophisticated control system in which the matching circuits are actively tuned during the shot in order to maintain a good match as the plasma evolves. Indeed, it has become important to find a way to keep the rf power high during magnetohydrodynamic (MHD) activity such as edge-localized modes and during transitions to $H$ modes. It would be a technical challenge to perform the computations described in this article so that the complete scattering matrix could be determined in the required time scale, perhaps just a few hundred microseconds, and a bigger challenge to implement our Newton's method tuning algorithm in that time. However, with advancing computer technology and perhaps some optimization of the algorithms we believe it would be possible.

\section{ACKNOWLEDGMENTS}

The authors wish to thank Professor Noah Hershkowitz and the staff of the Phaedrus group at the University of Wisconsin for their support in this work. In particular, they thank the people who built the Phaedrus-T machine; the late Jim Pew, David Brouchous, H. Y. Che, Greg Winz, Greg Kant, Bob Johnson, Jim DeKock, Sol Walsh, Paul Nonn, Steve Oliva, Bill Zimmerman, and others. This work was supported by U.S. Department of Energy Grant No. DE-FG0288ER53264.

${ }^{1}$ T. J. Wade, J. Jacquinot, G. Bosia, A. Sibley, and M. Schmid, Fusion Eng. Des. 24, 23 (1994).

${ }^{2}$ F. W. Baity, R. H. Goulding, D. J. Hoffman, P. M. Ryan, J. S. deGrassie, C. C. Petty, and R. I. Pinsker, Fusion Eng. Des. 24, 91 (1994).
${ }^{3}$ R. Majeski, P. H. Probert, T. Tanaka, D. Diebold, R. Breun, M. Doczy, R. Fonck, N. Hershkowitz, T. Intrator, G. McKee, P. Nonn, J. Pew, and J. Sorensen, Fusion Eng. Des. 24, 159 (1994).

${ }^{4}$ M. Bures, H. Brinkschulte, J. Jacquinot, K. D. Lawson, A. Kaye, and J. A. Tagle, Plasma Phys. Control. Nucl. Fusion 30, 149 (1988).

${ }^{5}$ M. Bures, J. Jacquinot, K. Lawson, M. Stamp, H. P. Summers, D. A. D'Ippolito, and J. R. Myra, Plasma Phys. Control. Nucl. Fusion 33, 937 (1991).

${ }^{6}$ P. U. Lamalle, G. Bell, V. P. Bhatnagar, M. Bures, B. Fechner, C. Gormezano, J. Jacquinot, A. Kaye, M. Lennholm, F. Nguyen, E. Righi, A Sibley, D. F. H. Start, M. Timms, and T. J. Wade, 22nd European Physical Society Conference on Controlled Fusion and Plasma Physics, Bournemouth, UK, July 1995 (European Physical Society, Geneva, 1995), p. II-329.

${ }^{7}$ H. Kazumi, K. Yoshioka, S. Kinoshita, T. Yamamoto, C. C. Petty, and M. Saigusa, 18th European Physical Society Conference on Controlled Fusion and Plasma Physics, Berlin, Germany, June 1991 (European Physical Soceity, Geneva, 1991), p. III-329.

${ }^{8}$ R. Majeski, P. Probert, N. Hershkowitz, P. Moroz, D. Diebold, T. Tanaka, T. Intrator, R. Breun, D. Brouchous, R. J. Fonck, M. Kishinevsky, G. McKee, P. Nonn, J. Sorensen, and M. Vukovic, Plasma Phys. Control. Nucl. Fusion Res. 1, 751 (1993).

${ }^{9}$ R. Majeski, P. Probert, P. Moroz, T. Intrator, R. Breun, D. Brouchous, H. Y. Che, J. R. DeKock, D. Diebold, M. Doczy, R. Fonck, N. Hershkowitz, R. D. Johnson, M. Kishinevsky, G. McGee, J. Meyer, P. Nonn, S. P. Oliva, J. Pew, J. Sorenson, T. Tanaka, M. Vukovic, and G. Winz, Phys. Fluids B 5, 2506 (1993).

${ }^{10}$ S. Wukitch, M. Vukovic, R. Breun, D. Brouchous, D. A. Diebold, M. Doczy, A. Elfimov, D. Edgell, N. Hershkowitz, T. Intrator, M. Kishinevsky, C. Litwin, P. Moroz, and P. Probert, Phys. Rev. Lett. 74, 2240 (1995).

${ }^{11}$ T. Intrator, P. Probert, S. Wukitch, M. Vukovic, D. Brouchous, D. Diebold, R. Breun, M. Doczy, D. Edgell, A. Elfimov, Hershkowitz, M. Kishinevsky, C. Litwin, P Moroz, P. Nonn, and G. Winz, Phys. Plasmas 2, 2263 (1995).

${ }^{12}$ D. F. H. Start, G. Bell, V. P. Bhatnagar, M. Bures, G. A. Cottrell, L.-G. Eriksson, B. Fechner, R. Goulding, C. Gormezano, A. Howman, J. Jacquinot, A. Kaye, P. Lamalle, F. Nguyen, E. Righi, F. Rimini, A. Sibley, A. C. C. Sips, B. J. Tubbing, T. Wade, and D. Ward, AIP Conf. Proc. 355, 7 (1996). 